\title{
Contrataciones iguales o inferiores a 8 UITs y el Control Interno en la Municipalidad Distrital Colquepata -2019
}

\author{
Eduardo Huallpa Gutierrez \\ Municipalidad Distrital de Colquepata - Cusco, Perú
}

\begin{abstract}
Resumen
La presente investigación tiene como objetivo central, describir cómo son las contrataciones iguales o inferiores a 8 UITs, establecer el nivel de evaluación de riesgo en las contrataciones iguales o inferiores a 8 UIT, y establecer la situación de las actividades de control en las contrataciones iguales o inferiores a 8 UIT en la Municipalidad Distrital Colquepata - 2019. La investigación es de tipo aplicada a nivel descriptivo, de diseño no experimental, y la muestra es no probabilística, se basa en expedientes de contrataciones de bienes y servicios. Como resultado se obtuvo que existe un cumplimiento parcial de la normativa de contrataciones menores a 8UITs; con respecto al nivel de evaluación de riesgo existe un regular nivel de procedimientos para reaccionar ante los acontecimientos adversos que puedan afectar la consecución de objetivos institucionales; finalmente las actividades de control a las contrataciones se dan de manera regular. Por último, se concluyó que en materia de contrataciones existe un control ínfimo en el cumplimiento de la directiva que establece las contrataciones menores a 8UIT, y esto repercute negativamente en el cumplimiento de objetivos establecidos por la entidad.
\end{abstract}

Palabras clave: control interno, contrataciones de bienes y servicios, cadena de abastecimiento, eficiencia y eficacia, objetivos institucionales.

\section{Contracts equal to or less than 8 UITs and Internal Control in the District Municipality Colquepata -} 2019 


\begin{abstract}
The main objective of this research is to describe how are the contracts equal to or less than 8 UITs, to establish the level of risk assessment in contracts equal to or less than 8 UITs, and to establish the situation of control activities in contracts equal to or less than 8 UITs in the District Municipality Colquepata - 2019. The research is applied at a descriptive level, non-experimental design, and the sample is non-probabilistic, based on records of procurement of goods and services. As a result, it was obtained that there is a partial compliance with the regulations for contracts under 8 ITUs; with respect to the level of risk assessment, there is a regular level of procedures to react to adverse events that may affect the achievement of institutional objectives; finally, the control activities for contracts are regularly carried out. Finally, it was concluded that in terms of contracting, there is very little control over compliance with the directive that establishes contracting of less than 8 ITUs, and this has a negative impact on compliance with the objectives established by the entity.
\end{abstract}

Keywords: internal control, procurement of goods and services, supply chain, efficiency and effectiveness, institutional objectives.

\title{
Contratos iguais ou inferiores a 8 UITs e Controlo Interno no Município Distrital de Colquepata - 2019
}

\begin{abstract}
Resumo
A presente investigação tem como objectivo central, descrever como são as aquisições iguais ou inferiores a 8 UIT, estabelecer o nível de avaliação de risco em aquisições iguais ou inferiores a 8 UIT, e estabelecer a situação das actividades de controlo em aquisições iguais ou inferiores a 8 UIT no Município Distrital Colquepata - 2019. A investigação é de tipo aplicado a nível descritivo, desenho não experimental, e a amostra é não probabilística, baseia-se em registos de aquisição de bens e serviços. Como resultado, foi obtido que existe uma conformidade parcial com os regulamentos de aquisições ao abrigo de 8 UIT; no que respeita ao nível de avaliação de risco, existe um nível regular de procedimentos para reagir a acontecimentos adversos que podem afectar a realização de objectivos institucionais; finalmente, as actividades de controlo de aquisições são dadas regularmente. Finalmente, concluiu-se que no domínio da contratação existe um controlo mínimo em conformidade com a directiva que estabelece a contratação de menos de 8 UIT, o que tem um impacto negativo no cumprimento dos objectivos estabelecidos pela entidade.
\end{abstract}

Palavras-chave: controlo interno, aquisição de bens e serviços, cadeia de fornecimento, eficiência y eficácia, objectivos institucionais 


\section{Introducción}

En el Perú existen alrededor de 1678 municipalidades distritales (INEI, 2019) y dentro de ella se encuentra la Municipalidad Distrital de Colquepata, la cual, al igual que otras entidades, contrata bienes y servicios menores a 8 UIT para el cumplimiento de sus objetivos institucionales, de acuerdo a lo establecido en el literal a) del artículo 5 de la ley de contrataciones del estado № 30225. Dicha normativa indica que están exentos de la aplicación de la ley las contrataciones cuyos montos sean iguales o inferiores a 8 unidades impositivas tributarias vigentes al momento de la transacción; sin embargo, están sujetos a supervisión del Organismo Supervisor de Contrataciones del Estado (OSCE). Esta forma de contratación permite adquirir bienes y servicios de manera más rápida y directa, a través de un procedimiento especial de contratación que no necesita someter a un procedimiento de selección en el Sistema Electrónico de Contrataciones del Estado.

Las contrataciones menores a 8 UITs se practica estableciendo los procedimientos de contrataciones a través de la aprobación de una directiva por parte de la Gerencia Municipal o Gerencia de Administración, y dicha directiva describe el modo que se debe seguir para las adquisiciones de bienes y la contratación de servicios hasta por el monto de 33,600.00 soles UIT 2019 equivalente a s/ 4,200.00 (Decreto Supremo $\mathrm{N}^{\circ}$ 298-2018-EF, 2018).

Por otro lado, el control interno comprende las acciones de cautela previa, simultánea y de verificación posterior que realiza la entidad sujeta a control con la finalidad con la gestión de sus recursos, bienes y operaciones se efectúe correcta y eficientemente (Ley № 27785, 2002).

El riesgo es inherente en toda labor o proceso más aun en la aplicación de recursos del Estado, riesgos propios de los sujetos que intervienen, intrínsecos a su existencia y características particulares de cada asunto (Charris \& Llamas, 2016); por lo tanto, se requiere evaluar, controlar y supervisar el proceso de contratación pública de tal forma que el riesgo residual sea menor.

Acotando a lo que es el control interno, Mendoza, García, Delgado y Barreiro (2018) señalan que la implementación de los mecanismos de control interno refuerza los sistemas administrativos vinculados al ciclo de gasto público, lo cual favorece en mejores plazos tanto en los procesos de compras, logística, almacenaje, entre otros.

Porconsiguiente, formulamos la siguiente interrogante: ¿cómo son las contrataciones iguales o inferiores a 8 UIT y el Control Interno en la Municipalidad Distrital Colquepata - 2019? Para ello hemos establecido como objetivo principal el describir la situación de adquisición de bienes y el control interno en la Municipalidad Distrital Colquepata - 2019. Y como objetivos específicos tenemos: a) establecer el nivel de evaluación de riesgo en las contrataciones iguales o inferiores a 8 UIT en la Municipalidad Distrital Colquepata - 2019; b) explicar la situación de las actividades de control en las contrataciones iguales o inferiores a 8 UIT en la Municipalidad Distrital Colquepata - 2019. 


\section{Metodología}

La metodología de la presente investigación se basa en un método de nivel descriptivo no experimental (Carrasco, 2005) porque busca describir la situación de adquisición de bienes y el control interno, establecer el nivel de evaluación de riesgo en las contrataciones iguales o inferiores a 8 UIT, y explicar la situación de las actividades de control en las contrataciones iguales o inferiores a 8 UIT en la Municipalidad Distrital Colquepata - 2019. Para ello se empleó las técnicas de encuesta y análisis documental. Asimismo, se utilizaron el cuestionario y la ficha de observación como instrumentos para la recolección de datos.

La población materia de estudio está comprendida por diez funcionarios, quienes están involucrados en las contrataciones, y por las personas que intervienen en el control previo, simultaneo y posterior de las adquisiciones menores a 8 UIT. Para ello se empleó el cuestionario como instrumento. Por otro lado, la población abarca los expedientes de contrataciones de bienes y servicios por importes menores 0 iguales a 8 UITs, con el análisis documental y la ficha de observación como técnicas e instrumentos de recolección de datos.

\section{Resultados}

De acuerdo a los datos recolectados a través de las técnicas e instrumentos empleados, tenemos los siguientes resultados tabulados:

\section{Tabla 1: Los procedimientos de las adquisiciones documentados comunicados de manera clara y precisa a los responsables}

\begin{tabular}{|c|c|c|}
\hline & Frecuencia & Porcentaje \\
\hline Si & 10 & $100,0 \%$ \\
\hline No & 0 & $0,0 \%$ \\
\hline No Sabe & 0 & $0,0 \%$ \\
\hline Total & $\mathbf{1 0}$ & $\mathbf{1 0 0 . 0 0 \%}$ \\
\hline
\end{tabular}

Fuente: Elaboración propia

El resultado señala que todas las personas que intervienen en el proceso de adquisiciones de bienes y contratación de servicios fueron comunicadas de manera clara y precisa respecto a los procedimientos establecidos en la directiva para las contrataciones iguales o inferiores a 8 UIT, en concordancia con los principios que rigen las contrataciones públicas. Fueron comunicados sobre las responsabilidades, indagación de mercado, las penalidades por mora, las especificaciones técnicas y los términos de referencia, entre otros. 


\section{Tabla 2: Proveedores de bienes y servicios que se dedican al rubro materia de contratación}

\begin{tabular}{|c|c|c|}
\hline & Frecuencia & Porcentaje \\
\hline Cumple & 8 & 33,3 \\
\hline $\begin{array}{c}\text { Cumple } \\
\text { Parcialmente }\end{array}$ & 15 & 62,5 \\
\hline No Cumple & 1 & 4,2 \\
\hline Total & $\mathbf{2 4}$ & $\mathbf{1 0 0 . 0}$ \\
\hline
\end{tabular}

Fuente: Elaboración propia

El resultado expone que, dentro del expediente de contratación, los proveedores que participaron para la provisión de bienes y servicios cumplen de manera parcial para atender lo requerido por la entidad, es decir, que no se dedican al rubro materia de contratación.

Tabla 3: RNP de proveedores

\begin{tabular}{|c|c|c|}
\hline & Frecuencia & Porcentaje \\
\hline Cumple & 6 & $25,0 \%$ \\
\hline $\begin{array}{c}\text { Cumple } \\
\text { Parcialmente }\end{array}$ & 12 & $50,0 \%$ \\
\hline No Cumple & 6 & $25,0 \%$ \\
\hline Total & $\mathbf{2 4}$ & $\mathbf{1 0 0 , 0} \%$ \\
\hline
\end{tabular}

Fuente: Elaboración propia

La directiva establecía que, para participar y proveer bienes o servicios a la entidad, estos debían acreditar con la ficha del Registro Nacional de Proveedores (RNP) tratándose de importes que superen 01 (una) Unidad Impositiva Tributaria o s/ 4,200.00. El resultado obtenido demuestra que solamente la cuarta parte de las contrataciones cumple el requisito establecido en la normativa, por otro lado, existe un cumplimiento parcial que bordea el cincuenta por ciento. 
Tabla 4: Control previo de órdenes de compra y de servicio

\begin{tabular}{|c|c|c|}
\hline & Frecuencia & Porcentaje \\
\hline Cumple & 3 & 12,5 \\
\hline $\begin{array}{c}\text { Cumple } \\
\text { Parcialmente }\end{array}$ & 6 & 25,0 \\
\hline No Cumple & 15 & 62,5 \\
\hline Total & $\mathbf{2 4}$ & $\mathbf{1 0 0 , 0}$ \\
\hline
\end{tabular}

Fuente: Elaboración propia

Del resultado obtenido se traduce que más de la mitad de los expedientes de contrataciones menores a 8 UITs no pasaron el control previo, tanto para el devengado como para el girado, lo cual se evidencia a través de la impresión del sello de control interno que certifica que el expediente fue evaluado antes de proceder para el pago respectivo.

Tabla 5: Mecanismos para implantar sistemas de alerta ante acontecimientos que influyan en la consecución de objetivos

\begin{tabular}{|c|c|c|}
\hline & Frecuencia & Porcentaje \\
\hline Si & 5 & $50,0 \%$ \\
\hline No & 4 & $40,0 \%$ \\
\hline No Sabe & 1 & $10,0 \%$ \\
\hline Total & 10 & $\mathbf{1 0 0 , 0} \%$ \\
\hline
\end{tabular}

Fuente: Elaboración propia

Con respecto a los mecanismos o procedimientos para implantar sistemas de alerta para reaccionar ante los acontecimientos o cambios rutinarios en la consecución de objetivos, se demuestra que está establecido en un nivel intermedio, pues cualquier evento adverso en las contrataciones podrían obstaculizar que la entidad logre los objetivos establecidos.

\section{Conclusiones}

De acuerdo a los resultados obtenidos, se concluye que los involucrados en las contrataciones menores a 8 UIT tiene pleno conocimiento debido a la experiencia 
que adquirieron a través del tiempo y por las inducciones que le brindó la entidad respecto a la directiva que guía dicha contratación, previamente aprobada por la gerencia municipal. Sin embargo, se coteja que no se observan eficientemente por parte de los involucrados en la gestión logística y en los requisitos necesarios que deben cumplir los participantes en la contratación de bienes y servicios. De esta manera, se encuentra un obstáculo que garantiza a la entidad poder cumplir con los objetivos planteados.

Por otro lado, respecto al control interno se concluye que la no revisión de los expedientes de contratación no garantiza que el bien se haya entregado en su totalidad o el servicio sea prestado en su totalidad. Asimismo, se corre el riesgo de que, pese a haber sido efectuada, esta se realice de manera extemporánea.

Finalmente, luego de tratar sobre los procedimientos que la entidad debe establecer para la detección de posibles riegos en los procesos de contratación de bienes y adquisición de servicios - y cómo estas a su vez influyen negativamente en la obtención de logros institucionales-, la Municipalidad Distrital de Colquepata instauró sistemas de alerta para detectar dichas adversidades. Sin embargo, estas no resultan suficientes para garantizar y cautelar los recursos del Estado.

\section{Recomendaciones}

- Implementar el área de control interno para garantizar que las contrataciones menores a 8 UITs cumplan las exigencias establecidas en la directiva, antes de efectuar el devengado y girado por las obligaciones contraídas por la entidad.

- El riesgo es inherente a cada trabajo o proceso, más aún tratándose de recursos del Estado, por lo que se recomienda a la gerencia de administración hacer cumplir de manera estricta lo establecido en la directiva con el fin de optimizar los recursos de la entidad y lograr los objetivos institucionales.

- Realizar una revisión posterior de los expedientes que contienen las contrataciones de bienes y servicios para luego reforzar y corregir los errores incurridos. Todo ello ayudará a la mejora de la gestión municipal.

\section{Referencias bibliográficas}

Carrasco, S. (2005). Metodología de la Investigación Científica. Lima: San Marcos.

Charris, J. \& Llamas, A. (2016). El riesgo inherente al proveedor como criterio preventivo en la contratación pública. Derecho del Estado, 37, 95-125. Recuperado de https://papers.ssrn.com/sol3/papers.cfm?abstract_id=2897631

Decreto Supremo № 298-2018-EF. Aprueban valor de la Unidad Impositiva Tributaria durante el año 2019. MEF (2018). 
INEI. (2019). Perú: Numero de Municipalidades 2019 y Población total 2017, según Departamento. Recuperado de https://www.inei.gob.pe/media/MenuRecursivo/ publicaciones_digitales/Est/Lib1653/resumen.pdf

Ley No 27785. Ley Orgánica del Sistema Nacional de Control y de la Contraloría General de la República. MEF (2002).

Mendoza,W.,García,T.,Delgado,M.\&Barreiro,I.(2018).El control internoysu influencia en la gestión administrativa del sector público. Dominio de las Ciencias, 4(4), 206240. Recuperado de https://dialnet.unirioja.es/servlet/articulo?codigo=6656251

Fecha de recepción: 22/04/2021

Fecha de aceptación: 31/05/2021 Correspondencia: huallpaeduardo@gmail.com 